\title{
Character Roles and Interaction in the DynaLearn Intelligent Learning Environment
}

\author{
Michael Wißner ${ }^{1}$, Wouter Beek ${ }^{2}$, Esther Lozano ${ }^{3}$, Gregor Mehlmann ${ }^{1}$, \\ Floris Linnebank ${ }^{2}$, Jochem Liem² ${ }^{2}$, Markus Häring ${ }^{1}$, René Bühling ${ }^{1}$, \\ Jorge Gracia ${ }^{3}$, Bert Bredeweg ${ }^{2}$, and Elisabeth André ${ }^{1}$ \\ 1 Human Centered Multimedia, Augsburg University, Germany \\ \{wissner, mehlmann, haering, buehling, andre\}@informatik. uni-augsburg.de \\ 2 Human-Computer Studies, University of Amsterdam, The Netherlands \\ \{w.g.j.beek, f.e.linnebank, j.liem, b.bredeweg\}@uva.nl \\ 3 Ontology Engineering Group, Universidad Politécnica de Madrid, Spain \\ \{elozano, jgracia\}@fi.upm.es
}

\begin{abstract}
In this paper we present the cast of pedagogical agents in the DynaLearn Intelligent Learning Environment. We describe the different character roles and how they interact with the learners. Our aim in using these characters is to increase the learners' motivation.
\end{abstract}

\section{Introduction}

Virtual characters have been utilized in various learning environments. Most of them feature a teacher-like character that interacts with the learner $[1,2,3]$. Some make use of a character that can be taught by the user [2]. Some systems feature a fully embodied agent that also communicates non-verbally through gestures [1]. Some feature more than one character [2], but they do not interact with one another. It has also been shown that a one-sided coverage of knowledge transfer or the employment of only a single educational role may either lead to satisfying learning success or motivation, but usually not both at the same time [4].

We therefore hypothesize that a combination of these features, implemented in an integrated set of educational characters may better leverage learning. Hence, in the DynaLearn approach we decided to integrate a whole cast of character roles into our learning environment. DynaLearn is an intelligent learning environment in which learners learn by expressing their conceptual knowledge through qualitative reasoning models [5].

\section{The Characters in DynaLearn}

As we delineated in [6], the characters in DynaLearn are cartoonish hamsters. Figure 1 shows each of the characters with a typical line of dialog with regard to the model depicted in the center. 


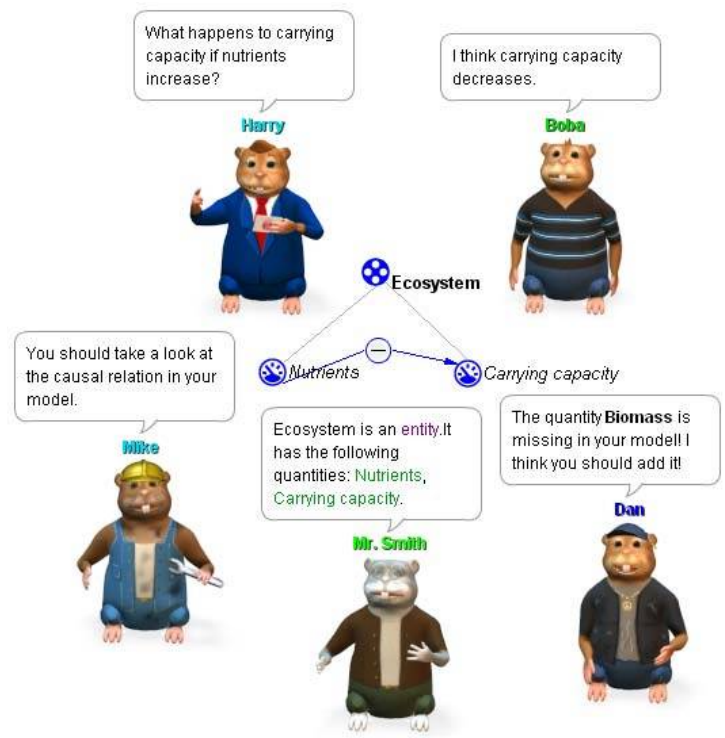

Fig. 1. The DynaLearn Characters (clockwise from top left): Quizmaster (QM), Teachable Agent (TA), Critic, Teacher, Mechanic

Teachable Agent: As the name implies, the TA has a knowledge representation that can be created by the learner. By testing the TA's understanding of the matter through questioning, the learner can evaluate his own presentation of the knowledge and detect mistakes when the TA does not answer as expected. Similar to [2], the interactions learners can perform with their TA in DynaLearn are: Ask (TA answers single questions), Explain (TA provides a step-by-step explanation of an answer) and Challenge (TA takes a quiz).

Mechanic: The task of the mechanic is to support learners in analyzing their model. Oftentimes, the simulation results of the model the learner created are not in line with the learner's expected outcome. An automated diagnostic component (based on [7]) detects these discrepancies, and identifies a minimum number of model components that caused this discrepancy. The mechanic is used to communicate these diagnosis results.

Teacher: In contrast to the mechanic, the teacher offers a more direct kind of help by communicating knowledge related to those aspects of the learning environment that learners can see and interact with. There are three such aspects: First, with respect to any one of the model ingredients, a "What is X?"-question can be posed. Second, with respect to each changing value in a model's simulation a "Why was X derived?"-question can be asked. Thirdly, a list of "How to $\mathrm{X}$ ?"-questions is constantly generated (where $\mathrm{X}$ is a task), based on the tasks that are available from the current context.

Quizmaster: The QM adds a playful element to the software and may be employed in a quiz directly with the human learner or with the learner's TA. The 
entertaining performance of QM and TA helps to point out flaws and verifies the correct parts of the learner's model. The question generator for the QM is based on the QUAGS question generator [8].

Critic: In contrast to the help provided by the mechanic or teacher characters, the critic's quality feedback about a learner's model is generated through an online repository of models, created by both other learners and experts [9]. Also, while the others are friendly and helpful, the critic is characterized as more strict and unforgiving.

\section{Conclusion}

We presented our approach to a cast of pedagogical agents, whose interactions with the learner offer a variety of services that help learners to verify and correct their models and conceptual knowledge, while motivating and engaging them at the same time.

\section{References}

1. Conati, C., Zhao, X.: Building and evaluating an intelligent pedagogical agent to improve the effectiveness of an educational game. In: Proc. of the 9th International Conference on Intelligent User Interfaces, pp. 6-13. ACM, New York (2004)

2. Biswas, G., Roscoe, R., Jeong, H., Sulcer, B.: Promoting self-regulated learning skills in agent-based learning environments. In: Proc. of the 17th International Conference on Computers in Education (2009)

3. Graesser, A.C., Person, N.K., Harter, D.: The Tutoring Research Group: Teaching tactics and dialog in autotutor. International Journal of AI in Education 12, 257 $279(2001)$

4. Kim, Y., Baylor, A.L.: PALS Group: Pedagogical agents as learning companions: The role of agent competency and type of interaction. Educational Technology Research and Development 54(3), 223-243 (2006)

5. Bredeweg, B., Linnebank, F., Bouwer, A., Liem, J.: Garp3 - workbench for qualitative modelling and simulation. Ecological Informatics 4(5-6), 263 (2009); Special Issue: Qualitative models of ecological systems

6. Mehlmann, G., Häring, M., Bühling, R., Wißner, M., André, E.: Multiple agent roles in an adaptive virtual classroom environment. In: Proc. of the 10th International Conference on Intelligent Virtual Agents, pp. 250-256. Springer, Heidelberg (2010)

7. de Koning, K., Breuker, J., Wielinga, B., Bredeweg, B.: Model-based reasoning about learner behaviour. Artificial Intelligence 117, 173-229 (2000)

8. Goddijn, F., Bouwer, A., Bredeweg, B.: Automatically generating tutoring questions for qualitative simulations. In: Proc. of the 17th International Workshop on Qualitative Reasoning, pp. 87-94 (2003)

9. Gracia, J., Liem, J., Lozano, E., Corcho, O., Trna, M., Gómez-Pérez, A., Bredeweg, B.: Semantic techniques for enabling knowledge reuse in conceptual modelling. In: Patel-Schneider, P.F., Pan, Y., Hitzler, P., Mika, P., Zhang, L., Pan, J.Z., Horrocks, I., Glimm, B. (eds.) ISWC 2010, Part II. LNCS, vol. 6497, pp. 82-97. Springer, Heidelberg (2010) 